\title{
Fungal keratitis caused by Beauveria bassiana: drug and temperature sensitivity profiles: a case report
}

\author{
Arisa Mitani ${ }^{1}$, Atsushi Shiraishi ${ }^{1,2^{*}}$, Hitoshi Miyamoto ${ }^{4}$, Atsuko Sunada ${ }^{5}$, Akiko Ueda ${ }^{5}$, Seishi Asari ${ }^{5}$, \\ Xiaodong Zheng ${ }^{1}$, Yasuaki Yamamoto ${ }^{1}$, Yuko Hara ${ }^{1}$ and Yuichi Ohashi, ${ }^{1,3}$
}

\begin{abstract}
Background: Beauveria bassiana is an entomopathogenic fungus and is a rare cause of keratitis. We present a case of fungal keratitis caused by B. bassiana that was diagnosed by in vivo confocal microscopy and in vitro corneal cultures. In addition, we determined the temperature- and drug-sensitivities of the isolated strain of B. bassiana.

Case presentation: A 59-year-old Japanese man with a 2-month history of keratitis was examined by slit-lamp biomicroscopy, in vivo confocal microscopy, and histology and cultures of corneal scrapings. The corneal scrapings were used to determine the minimal inhibitory concentrations of different antifungal drugs and also to determine the temperature-sensitivity. In vivo confocal microscopy and histological examinations showed filamentous fungal keratitis. The characteristics of the fungal growth indicated that the keratitis was caused by B. bassiana. The keratitis responded poorly to systemic and topical voriconazole and to natamycin ointment. However, it was resolved after changing the natamycin to micafungin combined with surgical debridement. The isolated strain was sensitive to itraconazole, miconazole, micafungin, voriconazole, and resistant to flucytosine and fluconazole. It was moderately sensitive to amphotericin $\mathrm{B}$, and natamycin. After 7 days in culture, the isolate grew small white colonies at $25^{\circ} \mathrm{C}$, very small colonies at $35^{\circ} \mathrm{C}$ and $37^{\circ} \mathrm{C}$.

Conclusion: The drug-sensitivity and temperature-sensitivity profiles of B. bassiana should be helpful in the treatment of $B$. bassiana keratitis. Therapeutic surgery may be helpful for mycotic keratitis poorly responsive to medical therapy alone.
\end{abstract}

Keywords: Fungal keratitis, Beauveria bassiana, In vivo confocal microscopy, Drug-sensitivity, Temperature sensitivity growth

\section{Background}

Beauveria bassiana is a fungus that is distributed worldwide. It can be isolated from soil, insects, and mites. It is an entomopathogenic fungi and is used as a biological control agent for insect pests as alternative supplements to chemical insecticides [1-3]. Although $B$. bassiana is considered to be non-pathogenic to vertebrates, a small number of patients with keratitis caused

\footnotetext{
* Correspondence: shiraia@m.ehime-u.ac.jp

'Department of Ophthalmology, Ehime University Graduate School of Medicine, Shitsukawa, Toon, Ehime 791-0295, Japan

2Department of Stem cell Biology, Shitsukawa, Toon, Ehime 791-0295, Japan Full list of author information is available at the end of the article
}

by $B$. bassiana have been reported [4-13]. We present a case of fungal keratitis caused by $B$. bassiana that was diagnosed by in vivo confocal microscopy and in vitro corneal cultures. In addition, we determined the temperature- and drug-sensitivities of the isolated strain of B. bassiana.

\section{Case presentation}

The patient was a 59-year-old Japanese man who noted a corneal opacity in his right eye in October, 2009. However, he did not seek treatment for the opacity for about two months. He visited a private ophthalmological clinic on December 11, 2009, because his right 
eye felt irritated and his vision had decreased. The patient was a farm worker, but did not use entomopathogenic fungi as a biological control agent. He had no systemic diseases and no signs or symptoms of immunosuppression during the clinical course. He was diagnosed with infectious keratitis and was treated with topical gatifloxacin and erythromycin eye ointment for two weeks. Because the signs and symptoms did not improve, he was referred to the Ehime University Hospital on December 28, 2009.

On the initial examination, his best-corrected visual acuity was 20/500 OD and 20/16 OS. Slit-lamp examination of the right eye showed a grayish stromal infiltrate with a dry texture and indistinct margins (Figure 1A). Examination of the cornea with in vivo confocal microscopy (HRT II-RCM; Heidelberg Engineering, Heidelberg, Germany) showed a mass of branching and interlocking white lines in the area of the infiltrate suggesting filamentous fungus keratitis (Figure 2A). Microscopic examination of corneal scrapings showed filamentous fungal hyphal fragments (Figure 2B).

Treatment was begun with oral voriconazole (400 mg/day) p.o., topical $0.1 \%$ voriconazole hourly, and natamycin eye ointment 5 times/day. Because the lesion did not respond despite the intensive antifungal therapy, the isolate from the corneal scrapings was sent to the First Laboratory in Medical Mycology Research Center, Chiba University for identification and to determine the minimal inhibitory concentrations (MICs) to different antifungal drugs (Table 1).

The corneal scrapings grew fungal colonies on Sabouraud dextrose agar and white colonies on potato dextrose agar (Figure 3A). Microscopic examination of the culture media stained with lactophenol cotton blue showed conidia in distinctive blue spore balls composed of a cluster of conidiogenous cells. The conidiogenous cells were short and ovoid and terminated in a narrow apical extension or rachis. The rachis was elongated after each conidium resulting in a long zig-zag extension. These features are characteristic of $B$. bassiana (Figure 3B).

The isolate was confirmed to be $B$. bassiana by DNA sequencing at the First Laboratory in Medical Mycology Research Center, Chiba University. The MIC of natamycin was determined to be $1 \mu \mathrm{g} / \mathrm{ml}$ by the modified agar plate dilution method at the Department of Infection Control, Osaka University Hospital [14-16].

After receiving the MIC results, the natamycin eye ointment (5 times/day) was changed to topical $0.1 \%$ micafungin (hourly) along with topical $0.1 \%$ voriconazole. During the next 3 months, surgical debridement was also performed 3 times and the infectious lesion slowly improved. Finally, the infectious lesion healed but with mild scar formation (Figure $1 \mathrm{C}$ ). His best-corrected visual acuity in his right eye improved to only 20/100 because of the corneal opacity and irregular astigmatism due to the surgical debridement.

To try to determine the cause of the slow clinical course and poor response to antifungal agents, the isolate was grown on Sabouraud dextrose agar at $25^{\circ} \mathrm{C}, 35^{\circ} \mathrm{C}$, and $37^{\circ} \mathrm{C}$ for up to 7 days at the Clinical Laboratory of Ehime University Hospital as described in detail [14]. The isolate grew very slowly, and no determination could be made at 5 days when most filamentous fungi would have already grown colonies. At 7 days, the isolate grew small white colonies at $25^{\circ} \mathrm{C}$, and very small colonies at $35^{\circ} \mathrm{C}$ and $37^{\circ} \mathrm{C}$ (Figure 4 ).

\section{Discussion}

The entomopathogenic fungi belonging to the genus Beauveria are rare pathogens in humans, and only 11 cases of keratitis due to Beauveria species have been reported [4-13]. Because of the limited number of cases, the clinical features of $B$. bassiana keratitis have not been determined. The course of our non-necrotic keratitis case was slow-growing, there was no clinical improvement after treatment with voriconazole even though voriconazole was the preferable antibiotic according to the

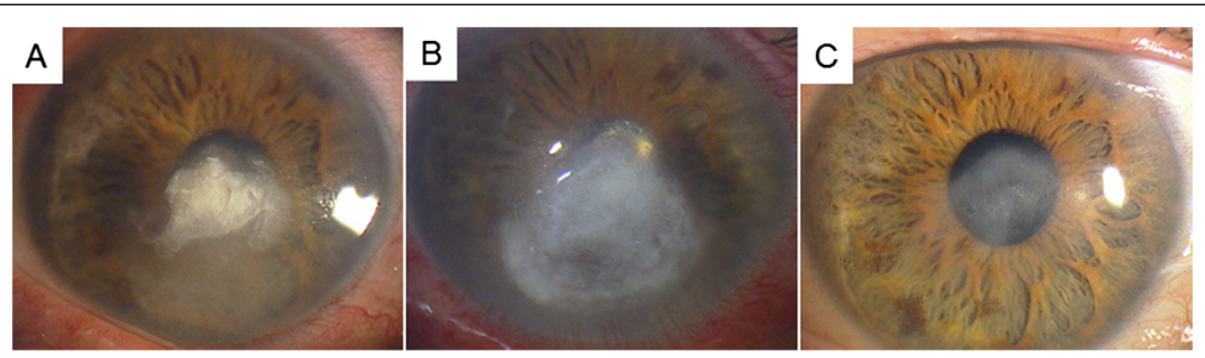

Figure 1 Slit-lamp photographs of the clinical course of Beauveria bassiana keratitis. A. Slit-lamp photograph taken at the initial examination showing a grayish stromal infiltrate with a dry texture and indistinct margins. B. Slit-lamp photograph 1 month after antifungal therapy with no improvement in the grayish stromal infiltrate. C. Slit-lamp photograph 10 month after treatment showing a scar with no infiltration into the stroma. 

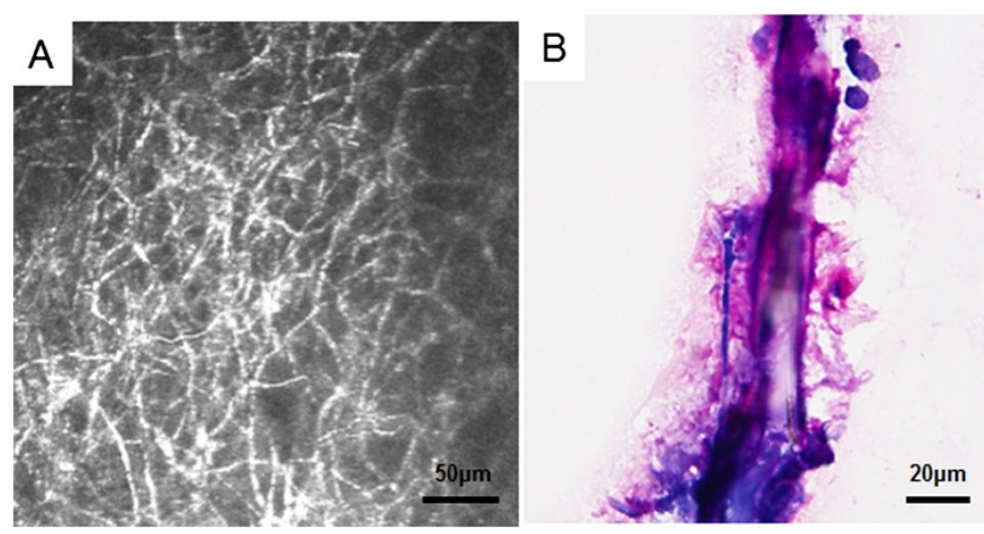

Figure 2 In vivo confocal microscopic image of Beauveria bassiana keratitis. A. A mass of interlocking and branching white lines in the area of the infiltrate indicating filamentous fungi. B. Microscopic examination of the corneal scrapings showing filamentous fungal hyphal fragments.

sensitivity tests. The cases of $B$. bassiana reported by Low et al., Kisla et al., and Tu et al., were also slowgrowing with late penetration into the corneal stroma which is consistent with our case $[6,7,12]$. In addition, the resistance to antifungal therapy was also consistent with our case [6-8,12].

Our antifungal susceptibility tests showed that the isolated strain was sensitive to micafungin and voriconazole and moderately sensitive to natamycin. There have been several reports on the drug sensitivity of B. bassiana. $\mathrm{Tu}$ et al. and Figueira et al. reported that both of their strains were sensitive to posaconazole and voriconazole but resistant to amphotericin. These sensitivities are similar to that of our strain of $B$. bassiana $[5,12]$. The results of Sonoyama et al. showed that the drug sensitivity of their Beauveria species was similar to our strain [11]. In addition, voriconazole was ineffective in both cases. Otherwise, the clinical course of the two cases was very

Table 1 Minimal inhibitory concentrations of different antifungal drugs

\begin{tabular}{lcc}
\hline Drug & RPMI & 0.5\% \\
\hline AMPH & 0.5 & 4 \\
$5-F C$ & $>64$ & $>64$ \\
FLCZ & 4 & 64 \\
ITZ & 0.03 & 0.03 \\
MCZ & 0.125 & 0.25 \\
MCFG & 0.125 & 0.06 \\
VCZ & 0.25 & 0.5 \\
& & MIC $(\mu \mathrm{g} / \mathrm{ml})$ \\
\hline
\end{tabular}

RPMI; Roswell Park Memorial Institute medium, YNB; yeast nitrogen base. $\mathrm{AMPH}$; amphotericin B, 5-FC; flucytosine, FLCZ; fluconazole, ITZ; itraconazole, $\mathrm{MCZ}$; miconazole, MCFG; micafungin, $\mathrm{VCZ}$; voriconazole, MIC; minimal inhibitory concentration. similar, viz., slow-growing, non-necrotic keratitis, and clinically resistant to voriconazole. However, the causative strain was sensitive to voriconazole. Sonoyama et al. also had a successful treatment with topical voriconazole, although they also treated the eye with topical miconazole and oral itraconazole which their strain was sensitive to $[11]$.

The results of the temperature sensitivity profile supported the microbiological results reported by Figueira et al. [5]. The temperature in the anterior chamber of the eye is generally $35^{\circ} \mathrm{C}$ or higher, and that of the

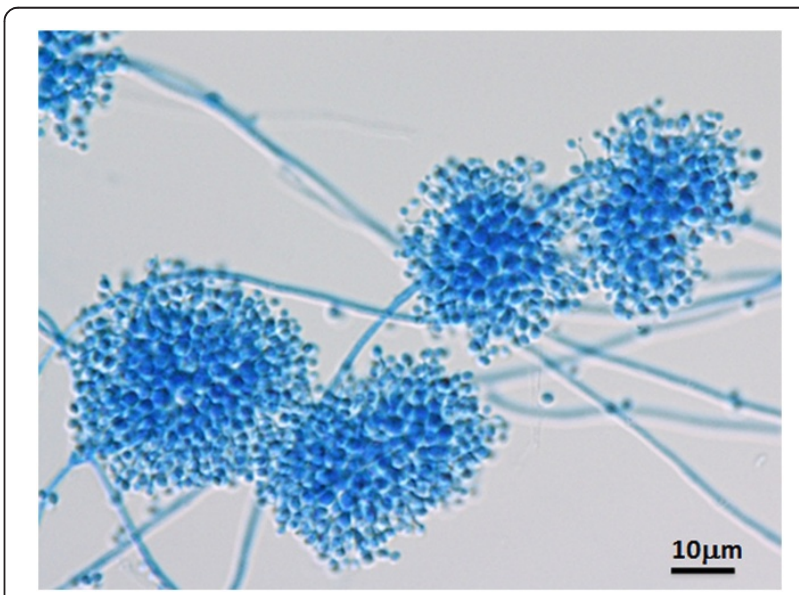

Figure 3 Microscopic examination of the culture media. Microscopic examination of the culture media stained with lactophenol-cotton blue shows conidia in distinctive blue spore balls composed of clusters of conidiogenous cells. The conidiogenous cells are short and ovoid and terminate in a narrow apical extension called a rachis. The rachis elongates after each conidium is produced resulting in a long zig-zag extension. These features are characteristic of B. bassiana. 


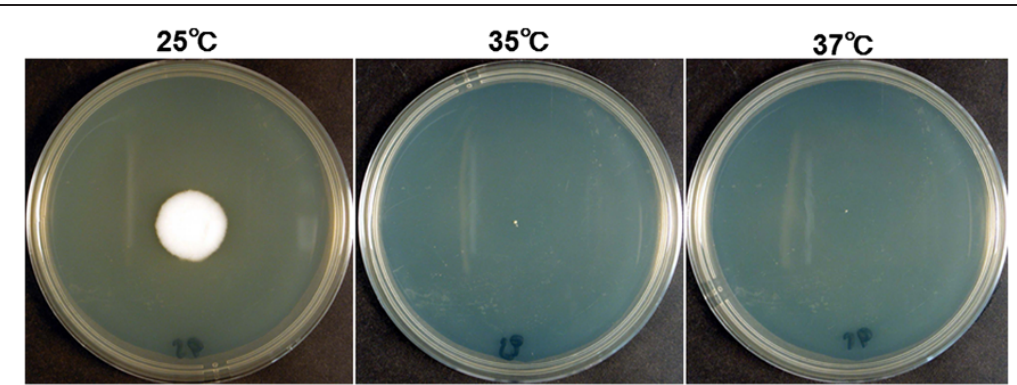

Figure 4 Fungal colonies grown on Sabouraud dextrose agar at different temperatures. The isolate grew small white colony at $25^{\circ} \mathrm{C}$, very small colony at $35^{\circ} \mathrm{C}$, and $37^{\circ} \mathrm{C}$ after 7 days. $(n=3)$.

corneal surface is $35^{\circ} \mathrm{C}$ or lower depending on the ambient temperature $[17,18]$. Thus, we suggest that the $B$. bassiana strain in our case could not grow at temperatures of $35^{\circ} \mathrm{C}$ or higher and thus did not penetrate into the corneal stroma. This temperature sensitivity might be one reason why $B$. bassiana is a rare pathogen in humans, and why the infections in humans are limited to the body surface such as the cornea.

Another reason for the infection of the cornea may be the ability of $B$. bassiana to produce chitinase which lyses not only chitin but also keratin and collagen. This would then enable it to adhere and penetrate the cornea [5,12]. B. bassiana has 3 different cell forms and can adhere to both hydrophobic and hydrophilic surfaces. This would enhance its adherence to the corneal surface along with the permissive avascular immune environment of the central cornea $[5,12]$. In addition, the slow-growing property may be one of the reasons why the initial intensive antifungal therapy seemed to be clinically ineffective, because voriconazole works by preventing fungi from producing ergosterol which is a component of fungal cell membranes, and ergosterol is necessary for fungi to proliferate. Thus, the initial treatment with voriconazole only appeared to be ineffective in such slow-growing fungi. Although the clinical course was very slow, the infectious lesion improved after changing the treatment from natamycin eye ointment to topical micafungin and surgical debridement.

Micafungin is a echinocandins which blocks fungal cell wall beta-glucan synthesis, thus the different actions of voriconazole and micafungin may have a synergetic effect. It is also possible that the surgical debridement might have worked in our case because it removed antigenic and infectious elements and necrotic tissues. This supports the results of earlier studies that surgical debridement or keratectomy is required for mycotic keratitis that respond poorly to medical therapy $[19,20]$. Further experiments on animals may be helpful in testing this hypothesis.

\section{Conclusions}

We report a case of $B$. bassiana keratitis. The drugsensitivity and temperature sensitivity profiles of $B$. bassiana should be helpful in diagnosing and treating B. bassiana keratitis. Therapeutic surgery may also be helpful for mycotic keratitis poorly responsive to medical therapy.

\section{Consent}

Written informed consent was obtained from the patient for publication of this Case Report and any accompanying images. A copy of the written consent is available for review by the Editor-in-Chief of this journal.

\section{Abbreviations}

B. bassiana: Beauveria bassiana; MIC: Minimal inhibitory concentration; RPMI: Roswell Park Memorial Institute medium; YNB: Yeast nitrogen base; AMPH: Amphotericin B; 5-FC: Flucytosine; FLCZ: Fluconazole;

ITZ: Itraconazole; MCZ: Miconazole; MCFG: Micafungin; VCZ: Voriconazol.

\section{Competing interests}

The authors declare that they have no competing interests.

\section{Authors' contributions}

$A M, A S, H M, A S, A U$, and $S A$ performed the examination and operation of the case. $X Z, Y Y, Y H$ and $Y O$ conceived of the design of this report. All authors read and approved the final manuscript.

\section{Acknowledgements}

The protocol for this study was approved by Institutional Review Board of Ehime University, No. 0612014. An informed consent for the examination was obtained from the subject, and the procedures used conformed to the tenets of the Declaration of Helsinki. The authors would like to thank Professor. Duco Hamasaki for editing the manuscript.

\section{Author details}

'Department of Ophthalmology, Ehime University Graduate School of Medicine, Shitsukawa, Toon, Ehime 791-0295, Japan. ${ }^{2}$ Department of Stem cell Biology, Shitsukawa, Toon, Ehime 791-0295, Japan. ${ }^{3}$ Department of Infectious Diseases Medicine, Ehime University Graduate School of Medicine, Shitsukawa, Toon, Ehime 791-0295, Japan. ${ }^{4}$ Department of Clinical Laboratory, Ehime University Hospital, Shitsukawa, Toon, Ehime 791-0295, Japan. ${ }^{5}$ Department of Clinical Laboratory, Osaka University Hospital, Suita, Osaka Prefecture 565-0871, Japan.

Received: 29 April 2014 Accepted: 22 September 2014 Published: 27 September 2014 


\section{References}

1. Samish M, Ginsberg H, Glazer I: Biological control of ticks. Parasitology 2004 129(Suppl):S389-S403.

2. St Leger RJ, Wang C: Genetic engineering of fungal biocontrol agents to achieve greater efficacy against insect pests. Appl Microbiol Biotechnol 2010, 85(4):901-907.

3. Wraight SP, Ramos ME, Avery PB, Jaronski ST, Vandenberg JD: Comparative virulence of Beauveria bassiana isolates against lepidopteran pests of vegetable crops. J Invertebr Pathol 2010, 103(3):186-199.

4. Ishibashi $Y$, Matsumoto $Y$, Takei $K$ : The effects of intravenous miconazole on fungal keratitis. Am J Ophthalmol 1984, 98(4):433-437.

5. Figueira L, Pinheiro D, Moreira R, Pinto E, Simoes J, Camisa E, Torrao L, Palmares J, Falcao-Reis F: Beauveria bassiana keratitis in bullous keratopathy: antifungal sensitivity testing and management. Eur J Ophthalmol 2012, 22(5):814-818.

6. Kisla TA, Cu-Unjieng A, Sigler L, Sugar J: Medical management of Beauveria bassiana keratitis. Cornea 2000, 19(3):405-406.

7. Low CD, Badenoch PR, Coster DJ: Beauveria bassiana keratitis cured by deep lamellar dissection. Cornea 1997, 16(6):698-699.

8. McDonnell PJ, Werblin TP, Sigler L, Green WR: Mycotic keratitis due to Beauveria alba. Cornea 1984, 3(3):213-216.

9. Oh JY, Lee MJ, Wee WR, Heo JW: A case of necrotizing sclerokeratitis and endophthalmitis caused by Beauveria bassiana. Jpn J Ophthalmol 2009, 53(5):551-553.

10. Pariseau B, Nehls S, Ogawa GS, Sutton DA, Wickes BL, Romanelli AM: Beauveria keratitis and biopesticides: case histories and a random amplification of polymorphic DNA comparison. Cornea 2010, 29(2):152-158

11. Sonoyama H, Araki-Sasaki K, Kazama S, Kawasaki T, Ideta H, Sunada A, Asari S, Inoue $Y$, Hayashi K: The characteristics of keratomycosis by Beauveria bassiana and its successful treatment with antimycotic agents. Clin Ophthalmol 2008, 2(3):675-678.

12. Tu EY, Park AJ: Recalcitrant Beauveria bassiana keratitis: confocal microscopy findings and treatment with posaconazole (Noxafil). Cornea 2007, 26(8):1008-1010.

13. Sachs SW, Baum J, Mies C: Beauvaria bassiana keratitis. Br J Ophthalmol 1985, 69(7):548-550.

14. Shiraishi A, Araki-Sasaki K, Mitani A, Miyamoto H, Sunada A, Ueda A, Asari S, Zheng $X$, Yamamoto Y, Hara Y, Ohashi Y: Clinical Characteristics of Keratitis Due to Colletotrichum gloeosporioides. J Ocul Pharmacol Ther 2011, 27(5):487-491

15. Institute CaLS: Reference Method for Broth Dilution Antifungal Susceptibility Testing of Filamentous Fungi; Approved Standard. CLSI Document 2002, 22(16):M38-A.

16. Institute CaLS: Methods for dilution antimicrobial susceptibility tests for bacteria that grow aerobically. CLSI document 2002, 23(2):M7-A7.

17. Kawasaki S, Mizoue S, Yamaguchi M, Shiraishi A, Zheng X, Hayashi Y, Ohashi Y: Evaluation of filtering bleb function by thermography. $\mathrm{Br} J$ Ophthalmol 2009, 93(10):1331-1336.

18. Morgan PB, Soh MP, Efron N, Tullo AB: Potential applications of ocular thermography. Optom Vis Sci 1993, 70(7):568-576.

19. Ansari Z, Miller D, Galor A: Current Thoughts in Fungal Keratitis: Diagnosis and Treatment. Curr Fungal Infect Rep 2013, 7(3):209-218.

20. Thomas PA, Kaliamurthy J: Mycotic keratitis: epidemiology, diagnosis and management. Clin Microbiol Infect 2013, 19(3):210-220.

\section{doi:10.1186/1756-0500-7-677}

Cite this article as: Mitani et al:: Fungal keratitis caused by Beauveria bassiana: drug and temperature sensitivity profiles: a case report. $B M C$ Research Notes 2014 7:677.

\section{Submit your next manuscript to BioMed Central and take full advantage of:}

- Convenient online submission

- Thorough peer review

- No space constraints or color figure charges

- Immediate publication on acceptance

- Inclusion in PubMed, CAS, Scopus and Google Scholar

- Research which is freely available for redistribution

Submit your manuscript at www.biomedcentral.com/submit 